\title{
Photovoltaic Solar Textiles ${ }^{\dagger}$
}

John I B Wilson 1,2,* and Robert R Mather 1,2

1 Power Textiles Limited, Upland House, Ettrick Road, Selkirk TD7 5AJ, UK; info@powertextiles.com

2 School of Engineering \& Physical Sciences, Heriot-Watt University, Riccarton, Edinburgh EH14 4AS, UK

* Correspondence: j.i.b.wilson@hw.ac.uk

+ Presented at the International Conference on the Challenges, Opportunities, Innovations and Applications in Electronic Textiles (E-Textiles 2019), London, UK, 12 November 2019.

Published: 4 December 2019

\begin{abstract}
Solar cells are an option for powering active electronics on textiles, but should be fully integrated to avoid compromising the flexibility and handle of the basic fabric. Photovoltaic (PV) cells conventionally use rigid silicon wafers but there are also thin-film options, although some are sensitive to moisture and oxygen, and others require processing temperatures outside the range of most flexible materials. The coating on textiles is also influenced by the fabric's texture, elasticity, and surface roughness. The demands of a flexible structure affect the choice of the other parts of PV cells, namely their electrical contacts and any encapsulation layers. The two alternative routes to a textile PV design are-(i) coat the fabric with successive layers needed to make a sandwich device, or (ii) coat individual yarns with these layers and then process them into a fabric, e.g., by weaving.
\end{abstract}

Keywords: photovoltaic; textile; flexible solar cell

\section{Introduction}

The two major sectors for photovoltaic (PV) textiles are firstly to power sensors and other electronics integrated into a wearable fabric, and then the large-scale use of solar power from awnings, sunshades, covers, and similar installations. At present there are no purely textile solar power products but many laboratory-scale versions that are vying for development into commercial applications.

After a brief explanation of the essential features of a photovoltaic cell and some of the options for the photoactive material, we discuss the attractive features of textile substrates and the limitations that are imposed on designing and fabricating textile-based PV's. The alternative methods for combining PVs and textiles are compared along with examples of how the restrictions of a textured, porous, flexible material are addressed without compromising the preparative conditions for PV's, in this meeting of two contrasting industries-semiconducting electronics and textiles. We shall concentrate on the approach that coats the PV layers on to a fully formed fabric.

Finally, we refer to some problems that yet remain or have only been partly addressed, that prevent the industrial manufacturing of these anticipated energy sources.

\section{Photovoltaic Cells}

Photovoltaic devices convert light into electricity without chemical change or requiring heat, which makes them long-lived unless they also comprise of any material that is prone to reaction with the ambient environment. The widely used silicon solar cells absorb visible sunlight to generate pairs of positive and negative charges and these are separated by a built-in electric field at a planar junction between p-type and n-type silicon-without this field the charges would be lost by recombination. All types of solar cell have this basic construction-a layered sandwich of active 
semiconductor between two electrical contacts. An exception is the dye sensitized solar cell, DSSC, that contains a liquid or paste electrolyte to support a reversible redox reaction and so is really a photoelectrochemical device with possible side reactions that can degrade performance. Other materials have different absorption spectra, output voltages and currents, and costs. For textile-based cells, it is the lower cost thin-film materials that are appropriate-amorphous silicon, CdTe, CIGS, organics (including polymers), and DSSC, and the newer perovskites and kesterites.

The performance of all solar cells may be assessed under standard test conditions $-1 \mathrm{~kW} \mathrm{~m}^{-2}$, the AM1.5 solar spectrum, and at $25^{\circ} \mathrm{C}$. The current and voltage that are delivered to an electrical load depend on the load's resistance-the maximum output power (the product of current and voltage) lies between the short circuit current (i.e., zero resistance, which does not harm the cells) and the open circuit voltage (i.e., infinite resistance). This is the maximum efficiency for the conversion of sunlight to electricity and is around $20 \%$ for rigid silicon cells and much less for all types of flexible cell. Interconnections between cells will increase the voltage (series connection) or current (parallel connection) as required.

\section{Textiles as Substrates}

The features that we associate with fabrics, such as drape, folding, elasticity, and texture, make it difficult to fabricate PV cells without some prior treatment. The demands of the electrical conductors and the semiconducting material require consideration of the porosity, flexibility, transparency, and stability of the textile. Whilst electrical conduction can be provided by metal or transparent conducting oxide coatings using a variety of physical and chemical methods, the photoactive semiconductors are more difficult to coat. Some require high temperatures or ultraviolet annealing, and some are deposited by electrical plasma in a low-pressure reactive gas. All constituent layers must be conformal and electrical conduction must be continuous across the area of the cell.

Although the two types of products, PV and textile, may be fabricated separately and then one attached to the other, this is not a true textile substrate as it hinders the fabric's behaviour and may compromise the design aesthetics. Hence there are two approaches that may be taken-either fabricate PV fibres and produce a textile from these whilst electrically connecting the many long, thin individual cells, or add the PV layers to a completed textile which has an uneven and mobile surface. Nonetheless, textile substrates offer a support that is lightweight and easily transported before unfurling. They also contain far less embedded energy than glass substrates-woven polyester has about one third to one quarter of the energy used in manufacturing glass [1].

The choice of textile material ranges from specialist polyimides to commodity polyester, depending on the restrictions of PV fabrication. For those PV devices that require high temperature synthesis or annealing, glass fibre may be the obvious choice. For finished fabrics, there are knitted, knotted, woven, and non-woven types-weaving offers many alternative constructions that are dimensionally stable but remain flexible, however it is a rugged process that can strip off coatings. Coating by the PV layers has a wide choice, ranging from liquid (dip coating, spin coating, spray coating, and printing) to gaseous (chemical vapour deposition, CVD, and plasma enhanced CVD) that may or may not be familiar to the textile industry [2].

\section{Textile Photovoltaics}

The first challenge in fabricating any PV cell is to provide an electrically conducting base that does not have a resistive barrier to the flow of charges from the cell. For cells that deliver significant currents only metals are used, whereas lower performing thin film cells may use less conducting transparent oxides (e.g., indium tin oxide, ITO, or aluminium zinc oxide, AZO). Conducting polymers are not sufficiently conducting on their own. Several researchers on fibre organic PV cells make use of PEDOT:PSS in combination with graphene and/or silver nanowire (Ag-NW) layers (e.g., [3]). These are sufficiently conducting and very flexible, although Ag-NW's will have to be protected against atmospheric corrosion if exposed and are sharp enough to penetrate adjacent layers. The roughness of woven textiles has been smoothed out by dip coating in a resin [4], which makes the 
textile rather stiff or by screen-printing liquid polyimide [5]. Alternatively, doctor blading a PEDOT:PSS layer before metallising provides a smooth surface and also an electrical path past any microcracks in the metal conductor.

Careful weaving of metallised polymer warp yarns and coated polymer photoanode weft yarns has provided an all-solid DSSC textile that has connections to the aligned weft electrodes to make strings of the small PV cells and yield a 1.3\% efficiency [6]. The more traditional liquid-based DSSC was the basis for a woven textile using warp and weft glass fibres with weft-aligned titanium photoanode and counter electrode wires, that were coated with electrode paste and annealed at 480 ${ }^{\circ} \mathrm{C}$ after weaving and then sealed into a pouch for electrolyte containment [7].

This high temperature annealing required for many DSSC's eliminates many common textile materials, but organic PV's offer more choice. Spray coating was used to cover the woven polyester cotton with all the layers for an organic PV, after planarizing the fabric with a screen-printed polyurethane layer [8]. These types of cells require effective encapsulation to prevent atmospheric oxidation of the photoactive polymer. They also need care with the Ag-NW electrodes as these can short circuit through adjacent thin layers - replacing one contact with a silver layer and optimizing the thicknesses of all layers gave a much improved efficiency of $1.23 \%$ [9]. By comparison, solution-processed organic PVs using Ag-NW's and spin-coated PEDOT:PSS doped with ethylene glycol (front electrode), PM6: IT-4F (active polymers), PFN-Br (interface layer), and Al (rear contact) on PET polyester were over $10 \%$ efficient showing what is achievable on a plastic sheet, albeit for only small $4 \mathrm{~mm}^{2}$ devices [10].

Our own technique uses the more traditional thin-film silicon, actually a silicon/hydrogen alloy (a-Si:H) on woven polyester fabric. The first step is to make the surface of the polyester into a continuous sheet by calendering and then to smooth it with a layer of PEDOT:PSS which is covered with evaporated or sputtered aluminium as the main electrical contact. On to this we coat three thin layers of a-Si:H by plasma enhanced CVD in a low pressure gas, which requires a temperature of 200 ${ }^{\circ} \mathrm{C}$, polyester-compatible. The cells are completed by a top contact of sputtered ITO. This sequence provides a PV coating that does not compromise the flexibility of the underlying textile and is strong enough to withstand bending and stretching [11]. In addition, unlike most photovoltaic materials (apart from perovskites and DSSC's), the colour may be tailored by altering the layer thicknesses as it arises from optical interference and so will change according to the viewing angle (Figure 1). The presence of small defects from too-thin or non-conformal layers reduces the efficiency to below $1 \%$ at present, but this is not a fundamental restriction of the technique.

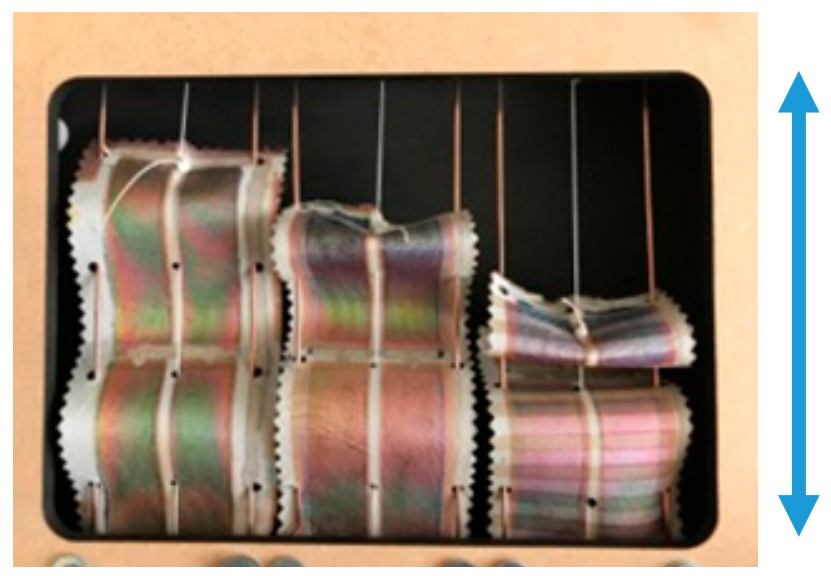

Figure 1. Amorphous silicon solar cells on woven polyester in a flexible demonstration jig. The arrow is $150 \mathrm{~mm}$ in length.

\section{Problems Remaining}

Efforts to reduce the roughness and discontinuity of textile surfaces have given some effective methods that do not impair the flexibility of textiles and a variety of materials offering effective electrical contacts. The basic structure of thin-film PV cells continues to be challenging as it requires 
thin conformal layers to provide high optical absorption without weakening the ability of the built-in field to separate photo-generated charges. Although a textured surface can reduce optical reflection losses and can scatter light into longer paths through the cell, it is difficult to lay down very thin coatings, one over the other, without some errors in layer separation. The issue of effective hermetic sealing, essential for the durability of some types of cells, has not been fully solved without compromising flexibility [12]. Finally, large area production of PV's on textiles lags way behind PV's on plastic film but all-solution processing offers Roll-to-Roll production for some types of cells [13]. Many applications await the successful outcome of current laboratory-scale development.

Author Contributions: Both authors have had an equal share in producing the material of this paper.

Funding: This research received no external funding.

Acknowledgments: We thank J\&D Wilkie, Kirriemuir, for woven polyester used for our photovoltaic coatings and former students at Heriot-Watt University who have worked on different topics relating to flexible photovoltaics, Adel Diyaf, Suzanne Jardine, Helena Lind, Artem Lukianov.

Conflicts of Interest: The authors declare no conflict of interest.

\section{References}

1. Lind, A.H.N.; Mather, R.R.; Wilson, J.I.B. Input energy analysis of flexible solar cells on textile. IET Renew. Power Gener. 2015, doi:10.1049/iet-rpg.2014.0197.

2. Mather, R.R.; Wilson, J.I.B. Fabrication of photovoltaic textiles. Coatings 2017, 7, 63-84, doi:10.3390/coatings7050063.

3. Wu, C.; Kim, T.W.; Guo, T.; Li, F. Wearable ultra-lightweight solar textiles based on transparent electronic fabrics. Nano Energy 2017, 32, 367-373, doi:10.1016/j.nanoen.2016.12.040.

4. Plentz, J.; Andra, G.; Pliewischkies, T.; Bruckner, U.; Eisenhawer, B.; Falk, F. Amorphous silicon thin-film solar cells on glass fiber textiles. Mater. Sci. Eng. B 2016, 204, 34-37, doi:10.1016/j.mseb.2015.11.007.

5. Liu, J.; Li, Y.; Yong, S.; Arumagam, S.; Beeby, S. Flexible printed monolithic-structured solid-state dye sensitized solar cells on woven glass fibre textile for wearable energy harvesting applications. Sci. Rep. 2019, 9, 1362, doi:10.1038/s41598-018-37590-8.

6. Zhang, N.; Chen, J.; Huang, Y.; Guo, W.; Yang, J.; Du, J.; Fan, X.; Tao, C. A wearable all-solid photovoltaic textile. Adv. Mater. 2016, 28, 263-269, doi:10.1002/adma.201504137.

7. Yun, M.J.; Cha, S.I.; Kim, H.S.; Seo, S.H.; Lee, D.Y. Monolithic-structured single-layered textile-based dye-sensitized solar cells. Sci. Rep. 2016, 6, 34249, doi:10.1038/srep34249.

8. Arumugam, S.; Li, Y.; Senthilarasu, S.; Torah, R.; Kanibolotsky, A.L.; Inigo, A.R.; Skabara, P.J;; Beeby, S.P. Fully spray-coated organic solar cells on woven polyester cotton fabric for wearable energy harvesting applications. J. Mater. Chem. A 2016, 4, 5561-5568.

9. Arumugam, S.; Li, Y.; Glanc-Gostkiewicz, M.; Torah, R.N.; Beeby, S.P. Solution Processed Organic Solar Cells on Textiles. IEEE J. Photovolt. 2018, 8, 1710-1715, doi:10.1109/JPHOTOV.2018.2871334.

10. Lei, T.; Peng, R.; Song, W.; Hong, L.; Huang, J.; Fei, N.; Ge, Z. Bendable and foldable flexible organic solar cells based on Ag nanowire films with $10.30 \%$ efficiency. J. Mater. Chem. A 2019, 7, 3737-3744, doi:10.1039/c8ta11293b.

11. Diyaf, A.G.; Mather, R.R.; Wilson, J.I.B. Contacts on polyester textile as a flexible substrate for solar cells. IET Renew. Power Gener. 2014, 8, 444-450, doi:10.3390/coatings7050063.

12. Uddin, A.; Upama, M.B.; Yi, H.; Duan, L. Encapsulation of Organic and Perovskite Solar Cells: A Review. Coatings 2019, 9, 65-82, doi:10.3390/coatings9020065.

13. Meng, X.; Zhang, L.; Xie, Y.; Hu, X.; Xing, Z.; Huang, Z.; Liu, C.; Tan, L.; Zhou, W.; Sun, Y.; et al. A General Approach for Lab-to-Manufacturing Translation on Flexible Organic Solar Cells. Adv. Mater. 2019, 31, 1903649-1903659, doi:10.1002/adma.201903649.

(C) 2019 by the authors. Submitted for possible open access publication under the terms and conditions of the Creative Commons Attribution (CC BY) license (http://creativecommons.org/licenses/by/4.0/). 